\title{
The Study on Multi-Attribute Decision-Making with Risk Based on Linguistic Variable*
}

\author{
Peide Liu $^{\dagger}$ \\ Information Management School, Shandong Economic University, No.7366,Erhuandong Road \\ Jinan 250014, P.R. of China \\ Xin Zhang \\ Information Management School, Shandong Economic University, No.7366,Erhuandong Road \\ Jinan 250014, P.R. of China \\ E-mail: zh_xin@sdie.edu.cn \\ www.sdie.edu.cn
}

Received: $03-09-2009$
Accepted: 15-10-2010

\begin{abstract}
An approach based on relative optimal membership degree is proposed to deal with multiple attribute decisionmaking (MADM) problems under risk with weight information unknown and attribute value as linguistic variable. Firstly, the operational laws of linguistic variable are introduced, and risk linguistic decision matrix is transformed into certain linguistic decision matrix by expectation value. Then, the ideal solution and negative ideal solution with linguistic variable are defined, and the attribute weight model is developed by relative optimal membership degree between alternatives and ideal solutions. In addition, the alternatives are ranked by relative optimal membership degree. Finally, illustrative example is provided to demonstrate the steps and effectiveness of the proposed approach.
\end{abstract}

Keywords: linguistic variable; risk decision; relative optimal membership degree; multiple attribute decisionmaking.

\section{Introduction}

Multiple attribute decision making (MADM) has been extensively applied to various areas such as society, economics, management, military and engineering technology. For example, Investment decision-making, project evaluation, economic evaluation, personnel evaluation, etc. The decision makers, evaluating some problems, often give the evaluation information in the form of linguistic term directly, such as good, medium good, poor, etc. For example, private morality, automobile performance etc. Therefore, the study on MADM problems with attribute value as linguistic variable has very important values on theoretical and practical application, and many achievements have been made $^{1-14}$. In addition, in the real decision-making process, the decision makers often face some problems, such as the uncertain environment and random variable as the attribute value, which make the decision makers not to determine the further status, but they can give the possible status, and quantify this randomness by setting up the probability distribution. This called multiple

* Received revised accepted

${ }^{\dagger}$ Corresponding author: Peide Liu. Email: Peide.liu@gmail.com, Tel:+86-531-82222188, 13964056802. The present address: No.7366, Erhuandong Road, Jinan 250014, Shandong Province, P.R. of China. The affiliation: Infprmation Management School, Shandong Economic University. 
attribute decision making with risk (MADMR) ${ }^{15}$. So the study on MADMR problems has the same important values on theoretical and practical applications. Since Zadeh (ref. 16) proposed the concept of a linguistic variable, the research on certain linguistic multiple attribute decision making problems has got rich achievements. Herrera et al. (refs. 1-3) presented the linguistic ordered weighted averaging (LOWA) operators to aggregate linguistic preference relations based on the ordered weighted averaging (OWA) operator defined by Yager (ref. 17) and the convex combination of linguistic labels defined by Delgado et al (ref. 18). Herrera and Herrera-Viedma (ref. 4) analyzed the problem of finding a solution set of alternatives from a collective linguistic preference relation, following two research directions: the choice functions and the mechanisms. Herrera et al. (ref. 5) introduced a framework to reach consensus in group decision making under linguistic assessments. Xu (ref. 19) proposed the linguistic order weighted geometric (LOWG) operators. Xu (ref. 20) studied the group decision making problems, in which all attribute weights, attribute values and the decision maker weights take the form of linguistic term, and the operational laws of the linguistic evaluation scales are defined and some new operators are developed, then a method based on the operators for multi-attribute group decision making under pure linguistic information is presented. $\mathrm{Xu}$ (ref. 21) presented an interactive procedure for linguistic multiple attribute decision making, in which the weight information is incomplete and the attribute values take the form of linguistic variable. Wei (ref. 22) studied the linguistic multiple attribute decision making problems, in which the attribute weights are completely unknown and the attribute values and the subjective preference values take the form of linguistic variable. The mathematical model is proposed to calculate the attribute weights, and the linguistic weighted arithmetic averaging (LWAA) operator is utilized to aggregate the linguistic decision-making information. Dong (ref. 23) studied the group decision-making problems with natural linguistic evaluation information, his method is that linguistic evaluation values are transformed into triangular fuzzy numbers, then the triangular fuzzy numbers are aggregated, finally, the best alternative is selected based on the aggregation results.

The research above is not studied the multiple attribute decision making problems with risk decision- making information, about the research on multiple attribute decision making problems with risk, some achievements have been gotten. At present, the main achievements are shown as follows: Yu (ref. 15) studies MADMR problems, in which the attribute weights are unknown and the attribute values are real numbers, and sets up the related mathematical model. Luo (ref. 24) studies MADMR problems in which the attribute weights are completely unknown and attribute values are interval numbers, and sets up two algorithms, grey fuzzy relationship method and two-basic-point method. Yao (ref. 25) and Konstantinos, et al (ref. 26) proposed the TOPSIS method for MADMR problems based on the continuous random variables. Rao and Xiao (ref. 27) proposed the method of dynamic hybrid multiple attribute decision making under risk, based on the unknown weight information and the attribute values which integrates with the precision number, interval number and linguistic fuzzy number. Jin, Zhang and Liu (ref. 27) proposed a rank approach based on projection model to deal with multiple attribute decision-making problems under risk and with attribute value as continuous random variable on bounded intervals. Firstly, risk decision matrix is normalized by density function, and weights of attributes are calculated based on exception value of random variable by using projection pursuit model and genetic algorithm. Then, through calculating weighted correlation coefficients between alternatives and ideal solutions, weighted grey correlation projection models on ideal solutions are developed by grey correlation projection method for every alternative, and alternatives are ranked by grey correlation projection value. Liu and Guan (ref. 28) proposed a grey correlation rank method to solve the problems of multiple attribute continuous decisionmaking under risk with weight unknown and attribute value as continuous random variable on bounded intervals. Firstly, risk decision matrix is normalized by density function, deviation between two random variables is defined by expectation value, and maximizing deviation rule is used to determine the weights of attributes. Then, ideal/negative ideal solutions are defined. Grey correlation degrees between alternatives and ideal/negative ideal solutions, and relative closeness coefficients are calculated. Furthermore, the alternatives are ranked by relative closeness coefficient of alternatives. 
The research above is not studied the MADMR problems with weight information unknown and attribute value as linguistic variable. With respect to these decision making problems, firstly, we transformed the risk linguistic decision matrix into certain linguistic decision matrix by expectation value. Then the attribute weights are determined and the alternatives are ranked by relative optimal membership degree. In order to do so, the remainder of this paper is organized as follows. In the next section, the definition of the multiple attribute decision making problems with risk is briefly introduced. In section 3, linguistic variable set and its extension are introduced; the ideal solution and negative ideal solution with linguistic variable are defined. The attribute weight model is developed by relative optimal membership degree between alternatives and ideal solutions, and the alternatives are ranked by relative optimal membership degree. In section 4, illustrative example is provided to demonstrate the steps and effectiveness of the proposed approach. In Section 5, we concluded the paper and give some remarks.

\section{Description of the Decision Making Problems}

The MADMR problems are represented as follows:
Suppose that $A=\left(a_{1}, a_{2}, \cdots, a_{m}\right)$ is the set of alternatives, and $C=\left(c_{1}, c_{2}, \cdots, c_{n}\right)$ is the set of attributes. Let the vector of attributes $W=\left(w_{1}, w_{2}, \ldots, w_{n}\right)$ be unknown, and $w_{j}$ represents the weight of attribute $c_{j}$, where $0 \leq w_{j} \leq 1, \quad \sum_{j=1}^{n} w_{j}=1$.Let $\Theta_{j}=\left(\theta_{1}, \theta_{2}, \ldots, \theta_{l_{j}}\right)$ be the possible status which belongs to the attribute $C_{j}$, and $p_{j}^{t}$ be the probability of the status $\theta_{t}$ occurred for the attribute $c_{j}$, where $0 \leq p_{j}^{t} \leq 1, \quad \sum_{t=1}^{l_{j}} p_{j}^{t}=1$. Let $x_{i j}^{t} \in S$ be the attribute value for the attribute $C_{j}$ and status $\theta_{t}$ with respect to the alternative $a_{i}$. Let $S$ be the linguistic assessment set, which is the ordered set with odd elements. For example, the linguistic assessment set $S$ has seven elements, then $S=$ (very poor, poor, medium poor, medium, medium good, good, very good). Then, we can evaluate the alternatives. (The MADMR data table is shown as Tab.1)

Table.1 risk multiple attribute decision-making table

\begin{tabular}{|c|c|c|c|c|}
\hline & $C_{1}$ & $C_{2}$ & $\cdots$ & $C_{n}$ \\
\hline & $\begin{array}{llll}\theta_{1} & \theta_{2} & \cdots & \theta_{l_{1}}\end{array}$ & $\begin{array}{llll}\theta_{1} & \theta_{2} & \cdots & \theta_{l_{2}}\end{array}$ & $\cdots$ & $\begin{array}{llll}\theta_{1} & \theta_{2} & \cdots & \theta_{l_{n}}\end{array}$ \\
\hline & $\begin{array}{llll}p_{1}^{1} & p_{1}^{2} & \cdots & p_{1}^{l_{1}}\end{array}$ & $\begin{array}{llll}p_{2}^{1} & p_{2}^{2} & \cdots & p_{2}^{l_{2}} \\
\end{array}$ & $\cdots$ & $\begin{array}{llll}p_{n}^{1} & p_{n}^{2} & \cdots & p_{n}^{l_{n}} \\
\end{array}$ \\
\hline$a_{1}$ & $\begin{array}{llll}x_{11}^{1} & x_{11}^{2} & \cdots & x_{11}^{l_{1}}\end{array}$ & $\begin{array}{llll}x_{12}^{1} & x_{12}^{2} & \cdots & x_{12}^{l_{2}}\end{array}$ & $\cdots$ & $\begin{array}{llll}x_{1 n}^{1} & x_{1 n}^{2} & \cdots & x_{1 n}^{l_{n}}\end{array}$ \\
\hline$a_{2}$ & $\begin{array}{lllll}x_{21}^{1} & x_{21}^{2} & \cdots & x_{21}^{l_{1}}\end{array}$ & $\begin{array}{llll}x_{22}^{1} & x_{22}^{2} & \cdots & x_{22}^{l_{2}}\end{array}$ & $\cdots$ & $\begin{array}{llll}x_{2 n}^{1} & x_{2 n}^{2} & \cdots & x_{2 n}^{l_{n}}\end{array}$ \\
\hline$\ldots$ & $\begin{array}{llll}\ldots & \ldots & \ldots & \ldots\end{array}$ & $\begin{array}{llll}\ldots & \ldots & \ldots & \ldots\end{array}$ & $\ldots$ & $\begin{array}{lllll} & \ldots & \ldots & \ldots & \ldots\end{array}$ \\
\hline$a_{m}$ & $x_{m 1}^{1} \quad x_{m 1}^{2} \cdots x_{m 1}^{l_{1}}$ & $x_{m 2}^{1} \quad x_{m 2}^{2} \quad \cdots \quad x_{m 2}^{l_{2}}$ & $\cdots$ & $x_{m n}^{1} \quad x_{m n}^{2} \cdots x_{m n}^{l_{n}}$ \\
\hline
\end{tabular}

\section{Evaluation Method}

In this section, with respect to MADM problems under risk with weight information unknown and attribute value as linguistic variable, a decision making method is proposed and detailed steps of this method are given. Firstly, linguistic variable set and its extension are introduced; the ideal solution and negative ideal 
solution with linguistic variable are defined. Then, attribute weight model is developed by relative optimal membership degree between alternatives and ideal solutions, and the alternatives are ranked by relative optimal membership degree.

\subsection{Linguistic assessment set and extended linguistic assessment set}

Let $S=\left(s_{0}, s_{1}, \cdots, s_{l-1}\right)$ be the linguistic assessment set with odd elements, where $l$ is the odd number, generally, $l$ is equal to $3,5,7$ or 9 . In this paper, let $l$ be 7 , then the assessment set is represented as $S=\left(s_{0}, s_{1}, s_{2}, S_{3}, s_{4}, s_{5}, s_{6}\right)=$ (very poor, poor, medium poor, medium, medium good, good, very good).

In these cases, it usually requires that $S_{i}$ and $S_{j}$ must satisfy the following additional characteristics:

(1) The set is ordered: $s_{i} \prec s_{j}$, if and only if $i<j$;

(2) There is the negation operator: $n e g\left(s_{i}\right)=s_{j}$, and $j=l-i$;

(3) Maximum operator: $\max \left(s_{i}, s_{j}\right)=s_{i}$, if $s_{i} \geq s_{j}$

(4) Minimum operator: $\min \left(s_{i}, s_{j}\right)=s_{i}$, if $s_{i} \leq s_{j}$

For each linguistic assessment set $S=\left(s_{0}, s_{1}, \cdots, s_{l-1}\right)$, the relationship between the element $S_{i}$ and its subscript $i$ is strict monotonic increasing $^{30}$.Therefore, we defined the function $f: s_{i}=f(i)$, obviously, the function $f(i)$ is the strict monotonic increasing function to the subscript $i$. In order to decrease the loss of the linguistic information, the discrete linguistic assessment set $S=\left(s_{0}, s_{1}, \cdots, s_{l-1}\right)$ is extended to the continuous set $\bar{S}=\left\{s_{\alpha} \mid \alpha \in R\right\}$, where the continuous linguistic assessment set $\bar{S}$ still satisfies the strict monotonicincreasing relationship.

The operational laws are defined as follows ${ }^{31}$ :

(1) $\beta s_{i}=s_{\beta \times i}$

(2) $s_{i} \oplus s_{j}=s_{i+j}$

(3) $s_{i} \oplus s_{j}=s_{j} \oplus s_{i}$
(4) $\lambda\left(s_{i} \oplus s_{j}\right)=\lambda s_{i} \oplus \lambda s_{j}$

(5) $\left(\lambda_{1}+\lambda_{2}\right) s_{i}=\lambda_{1} s_{i} \oplus \lambda_{2} s_{i}$

Definition $1^{31}$ : Let $S_{\alpha}$ and $S_{\beta}$ be two linguistic variables, and $s_{\alpha}, s_{\beta} \in \bar{S}$, then we defined the distance between $s_{\alpha}$ and $s_{\beta}$ as:

$$
d\left(s_{\alpha}, s_{\beta}\right)=|\alpha-\beta|
$$

\subsection{Transform risk decision-making matrix into certain decision-making matrix}

Based on the formula (1) to (5), we calculate the expectation value of each status with respect to the alternative in the risk decision-making table (shown in table.1), and combine to a certain linguistic decisionmaking matrix $Z$.

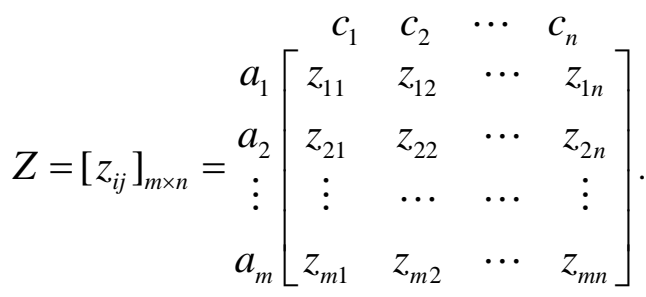

$$
\begin{aligned}
& \text { where } \quad Z_{i j}=\sum_{t=1}^{l_{j}} p_{j}^{t} x_{i j}^{t}
\end{aligned}
$$

\subsection{Positive Ideal Solution and Negative Ideal Solution}

Positive ideal solution (PIS) is the best alternative of all alternatives $a_{i}(i=1,2, \cdots, m)$, that is, each attribute value with respect to the PIS is the best value of this attribute with respect to all the alternatives in the decision matrix. While negative ideal solution (NIS) is the worst alternative, that is, each attribute value with respect to the NIS is the worst value of this attribute with respect to all the alternatives in the decision matrix.

$$
\begin{aligned}
& V^{+}=\left(v_{1}^{+}, v_{2}^{+}, \cdots, v_{n}^{+}\right) \\
& =\left(\max _{i}\left(z_{i 1}\right), \max _{i}\left(z_{i 2}\right), \cdots, \max _{i}\left(z_{i n}\right)\right) \\
& V^{-}=\left(v_{1}^{-}, v_{2}^{-}, \cdots, v_{n}^{-}\right) \\
& =\left(\min _{i}\left(z_{i 1}\right), \min _{i}\left(z_{i 2}\right), \cdots, \min _{i}\left(z_{i n}\right)\right)
\end{aligned}
$$




\subsection{Weight determined model}

The general weighted distance between each alternative $a_{i}$ and the ideal solution is defined as:

$$
\begin{aligned}
& D_{i}^{+}\left(a_{i}, V^{+}\right)=\sum_{j=1}^{n}\left[w_{j} d\left(v_{j}^{+}, z_{i j}\right)\right]^{2} \\
& D_{i}^{-}\left(a_{i}, V^{-}\right)=\sum_{j=1}^{n}\left[w_{j} d\left(v_{j}^{-}, z_{i j}\right)\right]^{2}
\end{aligned}
$$

where $d\left(v_{j}^{+}, z_{i j}\right)$ is the distance between the linguistic variable $v_{j}^{+}$and $z_{i j}$, and $d\left(v_{j}^{-}, z_{i j}\right)$ is the distance between the linguistic variable $v_{j}^{-}$and $Z_{i j}$.

The shorter the distance between each alternative $a_{i}$ and the positive ideal solution $V^{+}$is, the better the alternative is; the longer the distance between each alternative $a_{i}$ and the negative ideal solution $V^{-}$is, the better the alternative is.

Therefore, for each alternative $a_{i}$, we construct the programming model as follows:

$$
\begin{aligned}
& \min D_{i}^{+}\left(a_{i}, V^{+}\right)=\sum_{j=1}^{n}\left[w_{j} d\left(v_{j}^{+}, z_{i j}\right)\right]^{2} \\
& \text { s.t }\left\{\begin{array}{l}
\sum_{j=1}^{n} w_{j}=1 \\
w_{j} \geq 0
\end{array}\right. \\
& \max D_{i}^{-}\left(a_{i}, V^{-}\right)=\sum_{j=1}^{n}\left[w_{j} d\left(v_{j}^{-}, z_{i j}\right)\right]^{2} \\
& \text { s.t }\left\{\begin{array}{l}
\sum_{j=1}^{n} w_{j}=1 \\
w_{j} \geq 0
\end{array}\right.
\end{aligned}
$$

Due to each alternative is fair competition, and has none of the preference relationship, for formula (12), we can get the quadratic programming model as follows: $\min Z(w)=\sum_{i=1}^{m} D_{i}^{+}\left(a_{i}, V^{+}\right)=\sum_{i=1}^{m} \sum_{j=1}^{n}\left[w_{j}^{+} d\left(v_{j}^{+}, z_{i j}\right)\right]^{2}$

S.t $\left\{\begin{array}{l}\sum_{j=1}^{n} w_{j}^{+}=1 \\ w_{j}^{+} \geq 0\end{array}\right.$

Construct the Lagrange function:

$$
L=\sum_{i=1}^{m} \sum_{j=1}^{n}\left[w_{j}^{+} d\left(v_{j}^{+}, z_{i j}\right)\right]^{2}-2 \lambda\left(\sum_{j=1}^{n} w_{j}^{+}-1\right)
$$

Suppose that $\partial L / \partial w_{j}^{+}=0$, then:

$$
2 \sum_{i=1}^{m} d^{2}\left(v_{j}^{+}, z_{i j}\right) w_{j}^{+}-2 \lambda=0
$$

$$
\text { Namely, } w_{j}^{+}=\frac{\lambda}{\sum_{i=1}^{m} d^{2}\left(v_{j}^{+}, z_{i j}\right)}
$$

$$
\text { Since } \sum_{j=1}^{n} w_{j}^{+}=1 \text {, then } \sum_{j=1}^{n} \frac{\lambda}{\sum_{i=1}^{m} d^{2}\left(v_{j}^{+}, z_{i j}\right)}=1
$$

$$
\text { So, } \lambda=\left[\sum_{j=1}^{n}\left(\sum_{i=1}^{m} d^{2}\left(v_{j}^{+}, z_{i j}\right)\right)^{-1}\right]^{-1}
$$

Substitute the formula (17) into the formula (16), then:

$$
w_{j}^{+}=\left[\sum_{j=1}^{n}\left(\sum_{i=1}^{m} d^{2}\left(v_{j}^{+}, z_{i j}\right)\right)^{-1}\right]^{-1} / \sum_{i=1}^{m} d^{2}\left(v_{j}^{+}, z_{i j}\right)
$$

So we get: $W^{+}=\left(w_{1}^{+}, w_{2}^{+}, \cdots, w_{n}^{+}\right)$

Likewise, for formula (13), we can get the quadratic programming model as follows: 


$$
\begin{aligned}
& \max Z(w)=\sum_{i=1}^{m} D_{i}^{-}\left(a_{i}, V^{-}\right)=\sum_{i=1}^{m} \sum_{j=1}^{n}\left[w_{j}^{-} d\left(v_{j}^{-}, z_{i j}\right)\right]^{2} \\
& \text { s.t }\left\{\begin{array}{l}
\sum_{j=1}^{n} w_{j}^{-}=1 \\
w_{j}^{-} \geq 0
\end{array}\right.
\end{aligned}
$$

Construct the Lagrange function:

$$
L=\sum_{i=1}^{m} \sum_{j=1}^{n}\left[w_{j}^{-} d\left(v_{j}^{-}, z_{i j}\right)\right]^{2}-2 \lambda\left(\sum_{j=1}^{n} w_{j}^{-}-1\right)
$$

Then

$$
w_{j}^{-}=\frac{\left[\sum_{j=1}^{n}\left(\sum_{i=1}^{m} d^{2}\left(v_{j}^{-}, z_{i j}\right)\right)^{-1}\right]^{-1}}{\sum_{i=1}^{m} d^{2}\left(v_{j}^{-}, z_{i j}\right)}
$$

So we get $W^{-}=\left(w_{1}^{-}, w_{2}^{-}, \cdots, w_{n}^{-}\right)$

Suppose that $u_{i}$ is the relative optimal membership degree between the alternative $a_{i}$ and the PIS, then $1-u_{i}$ is the relative optimal membership degree between the alternative $a_{i}$ and NIS. The combined weighted distance between the alternative $a_{i}$ and ideal solutions which contain the PIS and NIS, namely, the square sum of the distance between the alternative $a_{i}$ and ideal solutions which contain the PIS and NIS, is defined as follows:

$$
\begin{array}{r}
f_{i}\left(u_{i}\right)=u_{i}^{2} \times\left(\sum_{j=1}^{n}\left[w_{j} d\left(v_{j}^{+}, z_{i j}\right)\right]^{2}\right) \\
+\left(1-u_{i}\right)^{2} \times\left(\sum_{j=1}^{n}\left[w_{j} d\left(v_{j}^{-}, z_{i j}\right)\right]^{2}\right)
\end{array}
$$

Obviously, for $u=\left(u_{1}, u_{2}, \cdots, u_{m}\right)$, the smaller the value of the function $f_{i}\left(u_{i}\right)$ is, the better the alternative $a_{i}$ is. So we can construct the multi-objective programming model:

$$
\min \left\{f(u)=\left(f_{1}\left(u_{1}\right), f_{2}\left(u_{2}\right), \cdots, f_{m}\left(u_{m}\right)\right)\right\}
$$

Satisfy the constraint conditions: $\left\{\begin{array}{l}\sum_{j=1}^{n} w_{j}=1, w_{j} \geq 0(j=1,2, \cdots, n) \\ 0 \leq u_{i} \leq 1, i=1,2, \cdots, m\end{array}\right.$

Calculate the derivative of $u_{i}$ for the formula (22):

$$
\frac{\partial f_{i}\left(u_{i}\right)}{\partial u_{i}}=0
$$

Simplified the formulas, then we get:

$$
u_{i}=\frac{D_{i}^{-}\left(a_{i}, V^{-}\right)}{D_{i}^{+}\left(a_{i}, V^{+}\right)+D_{i}^{-}\left(a_{i}, V^{-}\right)}
$$

$W^{+}$and $W^{-}$, determined by the formula (18)and (20) respectively, make that the alternative not only has the shortest distance from the PIS ,but also has the furthest distance from the NIS. Suppose that $W=\left(w_{1}, w_{2}, \cdots, w_{n}\right)$ be the weight vector. In order to get the biggest relative optimal membership degree, we construct the optimization model as follows:

$$
\begin{aligned}
& \max u_{i}=\frac{D_{i}^{-}\left(a_{i}, V^{-}\right)}{D_{i}^{+}\left(a_{i}, V^{+}\right)+D_{i}^{-}\left(a_{i}, V^{-}\right)} \\
& =\frac{\sum_{j=1}^{n}\left[w_{j} d\left(v_{j}^{+}, z_{i j}\right)\right]^{2}}{\sum_{j=1}^{n}\left[w_{j} d\left(v_{j}^{+}, z_{i j}\right)\right]^{2}+\sum_{j=1}^{n}\left[w_{j} d\left(v_{j}^{-}, z_{i j}\right)\right]^{2}} \\
& \text { s.t }\left\{\begin{array}{l}
\sum_{j=1}^{n} w_{j}=1 \\
w_{j} \in\left[\min \left(w_{j}^{-}, w_{j}^{+}\right), \max \left(w_{j}^{-}, w_{j}^{+}\right)\right]
\end{array}\right.
\end{aligned}
$$

Since the formula (26) is a nonlinear programming model, and it is difficult to solve the model for using the traditional method, we can utilize the genetic algorithms to solve this problem. Then we can get the weight vector $W=\left(w_{1}, w_{2}, \cdots, w_{n}\right)$

\subsection{Rank the order of alternatives}

Substituting the weight vector $W=\left(w_{1}, w_{2}, \cdots, w_{n}\right)$ into the formula (25), we can get the relative optimal membership degree $u_{i}$ between each alternative and the PIS. According to the relative optimal membership 
degree $u_{i}$, the ranking order of all the alternatives is determined. The more relative optimal membership degree $u_{i}$, the better the alternative is.

\section{Illustrate Example}

An enterprise will invest to construct a new factory. There are four alternatives. The four alternatives are assessed based on four attributes which are shown as follows: Direct efficiency $\left(c_{1}\right)$, Indirect efficiency $\left(c_{2}\right)$, Social efficiency ( $\left.C_{3}\right)$, Pollution $\operatorname{loss}\left(C_{4}\right)$. Direct efficiency ( $c_{1}$ )and Indirect efficiency $\left(c_{2}\right)$ are represented as four status, such as "very $\operatorname{good}\left(\theta_{1}\right)$ ", "good $\left(\theta_{2}\right)$ ", "medium( $\left.\theta_{3}\right)$ " and "poor $\left(\theta_{4}\right)$ ", and Social efficiency $\left(C_{3}\right)$,Pollution $\operatorname{loss}\left(C_{4}\right)$ are presented as three status, such as "very $\operatorname{good}\left(\theta_{1}\right) ", " \operatorname{good}\left(\theta_{2}\right)$ " and "medium $\left(\theta_{3}\right)$ ". The attributes of each alternative take the form of linguistic variable from linguistic variable set $S=\left(s_{0}, S_{1}, S_{2}, S_{3}, S_{4}, s_{5}, s_{6}\right)=$ (very poor, poor, medium poor, medium, medium good, good, very good). Based on the risk decision-making table of each attribute (shown as Table.2), we can get the best alternative.

The decision-making steps are shown as follows:

(1) Transform the risk decision-making matrix into a certain decision-making matrix:

$$
Z=\left[\begin{array}{llll}
\mathrm{S}_{4.1} & S_{4.2} & S_{3.8} & S_{3.6} \\
\mathrm{~S}_{4.2} & S_{4} & S_{3.1} & S_{3.3} \\
\mathrm{~S}_{3.6} & S_{3.9} & S_{3.9} & S_{3.7}
\end{array}\right]
$$

(2) Calculate the ideal solution of all alternatives:

$$
\begin{aligned}
V^{+} & =\left(s_{4.2}, s_{4.2}, s_{3.9}, s_{3.7}\right) \\
V^{-} & =\left(s_{3.6}, s_{3.9}, s_{3.1}, s_{3.3}\right)
\end{aligned}
$$

(3) Calculate the attribute weights:

$$
\begin{gathered}
W^{+}=(0.1517,0.4318,0.0864,0.3302) \\
W^{-}=(0.0992,0.6052,0.0536,0.2421) \\
W=(0.1342,0.5011,0.1050,0.2597)
\end{gathered}
$$

Table. 2 risk decision-making table of each attribute

\begin{tabular}{cccccccccccccccc}
\hline & \multicolumn{1}{c}{$c_{1}$} & \multicolumn{1}{c}{$c_{2}$} & \multicolumn{1}{c}{$c_{3}$} & \multicolumn{1}{c}{$c_{4}$} \\
\hline & $\theta_{1}$ & $\theta_{2}$ & $\theta_{3}$ & $\theta_{4}$ & $\theta_{1}$ & $\theta_{2}$ & $\theta_{3}$ & $\theta_{4}$ & $\theta_{1}$ & $\theta_{2}$ & $\theta_{3}$ & $\theta_{1}$ & $\theta_{2}$ & $\theta_{3}$ \\
\hline & 0.1 & 0.3 & 0.4 & 0.2 & 0.1 & 0.2 & 0.4 & 0.3 & 0.3 & 0.3 & 0.4 & 0.3 & 0.2 & 0.5 \\
\hline$a_{1}$ & $S_{5}$ & $S_{4}$ & $S_{5}$ & $S_{2}$ & $S_{4}$ & $S_{4}$ & $S_{3}$ & $S_{6}$ & $S_{5}$ & $S_{5}$ & $S_{2}$ & $S_{4}$ & $S_{2}$ & $S_{4}$ \\
$a_{2}$ & $S_{5}$ & $S_{3}$ & $S_{5}$ & $S_{4}$ & $S_{6}$ & $S_{3}$ & $S_{4}$ & $S_{4}$ & $S_{6}$ & $S_{3}$ & $S_{1}$ & $S_{2}$ & $S_{6}$ & $S_{3}$ \\
$a_{3}$ & $S_{4}$ & $S_{6}$ & $S_{2}$ & $S_{3}$ & $S_{1}$ & $S_{6}$ & $S_{5}$ & $S_{2}$ & $S_{4}$ & $S_{5}$ & $S_{3}$ & $S_{3}$ & $S_{4}$ & $S_{4}$ \\
\hline
\end{tabular}

(4) Calculate the weighted distance between each alternative and the ideal solutions:

$$
\begin{aligned}
& D^{+}=(0.0010,0.0279,0.0291) \\
& D^{-}=(0.0386,0.0090,0.0178)
\end{aligned}
$$

(5) Calculate the relative optimal membership degree:

$$
u=(0.9756,0.2439,0.3802)
$$

(6) Rank the order:

Based on the relative optimal membership degree, we can rank the order: $a_{1} \succ a_{3} \succ a_{2}$.

\section{Conclusions}


The MADMR problems are wildly used in various areas. This paper proposed a decision making method based on relative optimal membership degree for solving the MADMR problems in which the attribute weights are unknown and the attribute values are take the form of linguistic terms. Firstly, we transformed the risk linguistic decision matrix into certain linguistic decision matrix by expectation value. Then, the ideal solution and negative ideal solution with linguistic variable are defined, and the attribute weight model is developed by relative optimal membership degree between alternatives and ideal solutions. In addition, the alternatives are ranked by relative optimal membership degree. Then the attribute weights are determined and the alternatives are ranked by relative optimal membership degree. Finally, illustrative example is provided to demonstrate the steps and effectiveness of the proposed approach. This method is simple and easy to understand. This method constantly enriches and develops the theory and method of MADMR, and proposed a new idea for solving the MADMR problems. In the future, we shall continue working in the extension and application of the developed method to other domains.

\section{Acknowledgment}

This paper is supported by the Humanities and Social Sciences Research Project of Ministry of Education of China (No.09YJA630088), the Natural Science Foundation of Shandong Province (No. ZR2009HL022), the Social Science Planning Project Fund of Shandong Province (09BSHJ03), the Soft science project Fund of Shandong Province (2009RKA376), and Dr. Foundation of Shandong Economic University. The authors also would like to express appreciation to Editor Sonja Ruts, Editor-in-chief Da Ruan, and the anonymous reviewers for their very helpful comments on improving the paper.

\section{References}

1. F. Herrera, E. Herrera-Viedma, J.L. Verdegay, A sequential selection process in group decision making with linguistic assessment, Information Sciences 85(1995)223-239.

2. F. Herrera, L. Martinez, A 2-tuple fuzzy linguistic representation model for computing with words, IEEE Transactions on Fuzzy Systems 8 (2000) 746-752.

3. F. Herrera, E. Herrera-Viedma, J.L. Verdegay Direct approach processes in group decision making using linguistic OWA operators, Fuzzy Sets and Systems 79
(1996)175-190.

4. F. Herrera, E. Herrera-Viedma, Linguistic decision analysis: Steps for solving decision problems under linguistic information, Fuzzy Sets and Systems 115 (2000)67-82.

5. F.Herrera, E.Herrera-Viedma, J.L Verdegay, A model of consensus in group decision making under linguistic assessments, Fuzzy Sets and Systems 78(1996) 73-87.

6. F. Herrera, S. Alonso, F. Chiclana, E. Herrera-Viedma, Computing With Words in Decision Making: Foundations, Trends and Prospects. Fuzzy Optimization and Decision Making 8(4)(2009)337-364.

7. L. Martinez, M. J. Barranco, L.G. Perez, M.Espinilla , A Knowledge Based Recommender System with Multigranular Linguistic Information, International Journal of Computational Intelligence Systems, 1(3)(2008)225-236.

8. X. Li, D. Ruan, J. Liu, and Y. Xu, A linguistic-valued weighted aggregation operator to multiple attribute group decision making with quantative and qualitative information, International Journal of Computational Intelligence Systems, 1(3)(2008)274-284.

9. P.D. Liu, A novel method for hybrid multiple attribute decision making, Knowledge-based systems 22 (5)(2009) 388-391.

10. P.D. Liu, Multi-Attribute Decision-Making Method Research Based on Interval Vague Set and TOPSIS Method, Technological and economic development of economy 15(3)(2009) 453-463.

11. X. Zhang, P.D. Liu, Method for aggregating triangular fuzzy intuitionistic fuzzy information and its application to decision making, Technological and economic development of economy 2010,16(2)(2010)280-290

12. P.D. Liu, The Study on Venture Investment Evaluation Based on Linguistic Variable, ICIC Express Letters 4(1)(2010) 13-18.

13. F. Jin, P.D. Liu, X. Zhang, Third-party Logistics Performance Evaluation Research Based on Linguistic Variables and Grey Correlation Method, Journal of Information and Computational Science 6(4)(2009)1883-1890.

14. P.D. Liu, Z.L. Guan, Evaluation Research on the Quality of the Railway Passenger Service Based on the Linguistic Variables and the Improved PROMETHEE-II Method, Journal of Computers 4(3)(2009)265-270.

15. Y.B. Yu, B.D. Wang, P. Liu, Risky Multi-objective Decision-Making Theory and Its Application, Chinese Journal of Management Science 11(6) (2003)9-13.

16. L.A. Zadeh, The concept of a linguistic variable and its application approximate reasoning, Part 1, 2, and Part 3, Information Sciences 8 (3) (1975) 199-249; 8(4) (1975)301-357; 9(1) (1976) 43-58.

17. R.R. Yager, On ordered weighted averaging aggregation operators in multi-criteria decision making, IEEE Trans. Systems Man Cybernet. 18 (1988)183-190.

18. M. Delgado, J.L.Verdegay, M.A. Vila, on aggregation operations of linguistic labels, International Journal of 
Intelligent Systems, 8(1993) 351-370.

19.Z.S. Xu, A method based on linguistic aggregation operators for group decision making with linguistic preference relations, Information Sciences 166 (2004)19-30.

20.Z.S. Xu, On method of multi-attribute group decision making under pure linguistic information, Control and Decision 19(7) (2004)778-782.

21. Z.S. $\mathrm{Xu}$, An Interactive Procedure for Linguistic Multiple Attribute Decision Making with Incomplete Weight Information, Fuzzy Optimization and Decision Making 6(1) (2007) 17- 27.

22. G.W. Wei, W.D. Yi, Method of Linguistic Multiple Attribute Decision Making with Preference to Alternatives, Statistics and Decision (20)(2007)159-160.

23. Y.Q. Dong, L. Xiao, A Method of Group Decisionmaking based on Linguistic Assessment Information and Its Application, Fire Control and Command Control 33(5) (2008)97-100.

24. D. Luo, S.F. Liu, Research on grey multi-criteria risk decision-making method, Systems Engineering and Electronics 26(8) (2004) 1057-1060.

25. S.B. Yao, The TOPSIS Method for Multiple Attribute Decision Making with Continuous risk, Statistics and Decision (7) (2007)10-11.

26. Konstantinos Anagnostopoulos, Haris Doukas, John Psarras, A linguistic multi-criteria analysis system combining fuzzy sets theory, ideal and anti-ideal points for location site selection, Expert Systems with Applications 35(2008)2041-2048

27. C.J. Rao, X.P. Xiao, Method of grey matrix relative degree for dynamic hybrid multi-attribute decision making under risk, Systems Engineering and Electronics 28(9) (2006)1353-1357.

28. F. Jin, X. Zhang, P.D. Liu, Method For Multiple Attribute Decision-Making With Continuous Random Variable Under Risk Based On Projection Model, Mathematical And Computational Application 15(3)(2009)394-403.

29. P.D. Liu, Z.L. Guan, Research on Multiple Attribute Decision-Making under Risk with Continuous Random Variable and Weight Unknown, Systems Engineering and Electronics 31(9)(2009):2133-2137.

30.Z.S. Xu, A note on linguistic hybrid arithmetic averaging operator in multiple attribute group decision making with linguistic information, Group Decision and Negotiation 15(6) (2006)593- 604.

31.Z.S. Xu, Goal programming models for multiple attribute decision making under linguistic setting, Journal of Management Sciences in China 9(2) (2006)917. 